\title{
Evaluation of inhalation technique in patients using a dry powder device (DPI) at chest clinic in Dhulikhel Hospital - Kathmandu University Hospital, and the effect of patient education on it
}

\author{
Authors: Sudeep Shrestha, Sandhya Shrestha, Maun Ranjan Baral, Sambhawana Bhandari, Swati Chand, \\ Rajendra Tamrakar, Ram Kumar Mehta, Nirish Vaidya, Bijaya Krishna Prajapati, Satya Shrestha, Sherima \\ Pradhan and Prashiddha Dhakal
}

\section{Introduction}

The mainstay of chronic obstructive pulmonary disorder (COPD), asthma and asthma-COPD overlap syndrome (ACOS) treatment is by inhalational medication(s). Delivery of drugs directly into the airways, achieving higher local concentrations with significantly less risk of systemic side effects are the major advantages of the therapy. ${ }^{1}$ The delivery of inhaled medication in the airway tract is dictated by a complex interaction between the device, the aerosol formulation and patients' inhalation technique. ${ }^{2}$ The correct use of an inhaler device involves a series of steps, which need to be performed. Failing to perform one or more steps correctly can substantially reduce delivery and effectiveness, leading to poor disease control. ${ }^{1}$

Previous studies have reported that up to $94 \%$ of the patients used incorrect method of inhalation of the medication. ${ }^{3-5}$ The factors associated with poor inhalational technique include age, ${ }^{6,7}$ sex, ${ }^{8}$ education level ${ }^{6}$ and severity of obstruction. ${ }^{6}$ Improvement of patient's inhalation technique could be attained by educating the patients about proper use of device being used. Poor inhalation techniques are associated with decreased medication delivery and poor disease control in chronic lung diseases. The data in regards to the proportion of patients correctly using inhalation devices and the effect of patient education is currently lacking in Nepalese population.

This study hence intended to analyse the prevalence of patients using inhalation devices via incorrect technique and access the adherence of patients to correct inhalation technique when taught along with their subjective improvement of symptoms.

\section{Materials and methods}

This was a one group pre-test/post-test study design conducted in Dhulikhel Hospital - Kathmandu University Hospital, Dhulikhel, Nepal from March 2017 to May 2017. Patients presenting to the Chest Clinic in Dhulikhel Hospital, who were diagnosed with

Authors: Dhulikhel Hospital - Kathmandu University Hospital having either COPD, asthma or ACOS and using inhalational bronchodilators in the form of dry powder inhalers (DPI) via Rotahaler were included in the study.

The inclusion criteria were age $>18$ years, patients diagnosed of having of asthma or COPD or ACOS and those using dry powder inhaler (DPI) therapy with Rotahaler device. On the other hand, exclusion criteria included patients newly started on dry powder inhalation therapy or patients who had recently received face-to-face training program on inhalation technique within the past one month, patients in acute exacerbation and patients failing to give consent.

Enrolled patients were assessed for inhalation technique at their routine medical (pre-training) visit by a physician in the chest clinic. The use of each dry powder inhaler device (Rotahaler) was evaluated in a practical manner by asking the patients to demonstrate their inhalation technique. They were asked to mention each step as they performed them. An observation checklist measuring essential steps required for adequate drug delivery for Rotahaler as instructed by the manufacturer and from previous studies was developed. The number of required steps is seven:

1. Insert the Rotacap capsule, with transparent end first, into the raised square hole of the Rotahaler.

2. Press the Rotacap firmly, such that the top end of the Rotacap is level with the top of the hole.

3. Rotate the base of the Rotahaler device, such that the separated two ends of the Rotacap can be seen through the transparent body of the Rotahaler.

4. Breathe out slowly, away from the device mouthpiece.

5. Grip the mouthpiece gently between the teeth, seal the lips around it tight and take a deep breath.

6. Tilt the head slightly backwards and breathe in through the mouth as deeply as possible.

7. Remove the device from the mouth, holding the breath for as long as the patient can do it comfortably, before breathing out.

Correctness of the seven-step inhalation technique was measured by giving a score of 0 for incorrect action and 1 for correct action. Each of the seven steps were scored 1 or 0 , giving a total score of $0-7$. The total scores were then calculated. It was considered unlikely that a significant amount of medicine would be inhaled 
when one or more errors were made during these necessary steps. In these cases, the inhalation technique was defined as incorrect. The physician observed each step of inhalation technique and recorded each incorrect step. After assessment, instructions were given with demonstrations regarding the correct use of Rotahaler until the patient could use the device correctly. One month later (post-training visit), all patients were re-evaluated with regard to their inhalation technique.

Informed consent was taken from patients and the study was approved by the Institutional Review Committee of Dhulikhel Hospital - Kathmandu University Hospital (No. 73/17) and registered in ClinicalTrials.gov Identifier: NCT03275935.

Descriptive and inferential statistical analyses were used for the evaluation of data using Statistical Package for Social Sciences (SPSS 21.0). Continuous data with normal distribution were expressed as mean \pm standard deviation. Categorical data were described by absolute number and percentage of subjects per category. The potential risk factors for an incorrect inhalation technique were calculated via univariable logistic regression analysis. The results were displayed as odds ratios with $95 \%$ confidence intervals. Comparison percentages of incorrect inhalation technique between pre-training and post-training visits within groups were analysed using the Chi-square test. Paired t-tests were used for paired data comparison. Statistical significance was accepted at $p=0.05$.

\section{Results}

A total of 307 patients were included in the study. Patient demographics and clinical characteristics are presented in Table 1. More than half of the patients $(55 \%)$ were female. The most common diagnosis was COPD (69.1\%), followed by asthma (20.8\%) and ACOS (10.1\%). Nearly two-thirds of them (59.9\%) had a low level of education; ie had not completed their Grade 5).

More than half (58.3\%) of the patients were not given instruction on inhalation technique with a DPI device in the last 12 months. Upon examination of the device, six ( $2 \%$ ) participants were found to have non-functional device which was either clogged or broken. Three (1\%) participants were found to be taking the Rotacap orally.
The percentage of correctly performed steps before and after the training is shown in Table 1. Initially, $88.6 \%$ of the participants performed one or more of the steps incorrectly. Step 4 (Breathe out slowly, away from the device mouthpiece before inhalation) was the most frequently incorrectly performed step $(71.3 \%$ ) followed by step 7 (Remove the device from the mouth, holding the breath for as long as the patient can do it comfortably, before breathing out), which was $69.7 \%$.

Thirty-five patients who performed all the steps of inhalation correctly, along with 18 who did not attend follow up, were excluded from post-training analysis. Hence at the post-training visit, 254 of patients performed the DPI inhalation demonstrations and had their inhalation techniques reassessed. Overall, the proportion of patients performing incorrect steps of inhalation was $67.4 \%$. Assessment of formal training resulted in a statistically significant increase in inhalational score before and after training among the patients $(4.24 \pm 1.28$ pre-training and $6.07 \pm 0.81$ posttraining, $p<0.001)$

On bivariate analysis, incorrect inhalation technique was found to be significantly associated with low education level ( $<5$ th grade), age (>60yrs), and $\left(\mathrm{FEV}_{1}<50 \%\right)$.

Comparing the two visits, the patients responded to their subjective improvement in their symptom control. Almost three-quarters $(74.01 \%)$ of patients reported that they had had improvement in their symptoms after being taught the correct technique. Of these, 101 (39.8\%) reported that they had partial improvement; 58 (22.8\%) had marked improvement and 29 (11.4\%) had negligible improvement.

\section{Discussion}

This study shows that $88.6 \%$ of patients using dry powdered inhaler (DPIs) devices used their inhaler incorrectly by performing at least one essential step for drug delivery in the wrong way. The figure is comparable with previous studies. ${ }^{3-5}$ However, a study carried out in the same hospital in 2009 , revealed that $63 \%$ of the patients with COPD used their Rotahaler device incorrectly. ${ }^{9}$ The difference in the figures could be attributed to the fact that only outpatients were enrolled in our study, whereas both inpatient and outpatients were part of the 2009 study. Out of the seven steps,

Table 1. Results of inhalation steps before and after training

\section{Step Step details}

1 Insert the Rotacap capsule, with transparent end first, into the raised square hole of the Rotahaler.

2 Press the Rotacap firmly, such that the top end of the Rotacap is level with the top of the hole.

3 Rotating the base of the Rotahaler device, such that the separated two ends of the Rotacap can be seen through the transparent body of the Rotahaler.

$4 \quad$ Breathe out slowly, away from the device mouthpiece.

5 Grip the mouthpiece gently between the teeth, holding seal the lips around it tight and take a deep breath.

6 Tilt the head slightly backwards and breathe in through the mouth as deeply as possible.

7 Remove the device from the mouth, holding the breath for as long as the patient can do it comfortably, before breathing out.

$\begin{array}{llll}\begin{array}{l}\text { Visit 1 } \\ \text { correct, } \\ \mathbf{n}(\%)\end{array} & \begin{array}{l}\text { Visit 1 } \\ \text { incorrect, } \\ \mathbf{n}(\%)\end{array} & \begin{array}{l}\text { Visit 2 } \\ \text { correct, } \\ \mathbf{n}(\%)\end{array} & \begin{array}{l}\text { Visit 2 } \\ \text { incorrect, } \\ \mathbf{n}(\%)\end{array} \\ 304(99.0) & 3(1) & 254(100) & 0(0) \\ 300(98.4) & 7(2.3) & 252(99.2) & 2(0.8) \\ 279(91.5) & 28(9.1) & 253(99.6) & 1(0.4) \\ 88(28.9) & 219(71.3) & 205(80.7) & 48(18.9) \\ 159(52.1) & 148(48.2) & 231(90.9) & 23(9.1) \\ 150(49.2) & 157(51.1) & 213(83.9) & 41(16.1) \\ 93(30.5) & 214(69.7) & 135(53.1) & 119(46.9)\end{array}$


the most common error was made while 'breathing out slowly away from the mouthpiece before inhalation' and 'removing the device from mouth and holding the breath for as long as the patient comfortably could, before breathing out'. These errors were also consistent with the errors found in earlier studies. ${ }^{9-11}$ These high rates of poor DPI usage may be a result of inadequate training by medical professionals on the appropriate use of inhalation devices. Previous studies have shown that medical professionals, including physicians, nurses and pharmacists, may not be acquainted with appropriate device handling. ${ }^{12}$

The study also revealed a significant improvement in symptom control after face-to-face training.

This study confirms a significant increase in the percentage of improvement in inhalation technique after demonstrations and training. However, although the inhalation technique significantly improved post-training in follow up, the percentage of those using their device in a correct manner was still under $50 \%$. These outcomes advocate that patients should bring their inhaler devices to each appointment. Moreover, given that inhalation technique can deteriorate again after education, inhaler technique must be rechecked, and education must be regularly reinforced to maintain correct technique. ${ }^{13}$ Regular review and reinforcement of correct inhalation technique is essential for effective inhaler use.

Our study is one of the first in Nepal to evaluate the prevalence of patients using inhalation devices via incorrect technique and assess the adherence of patients to correct inhalation technique when taught. It confirms a significant improvement in inhalation technique after a simple face-to-face demonstration and training about the appropriate use of the device. In addition, the proper teaching translated well in terms of better symptom control.

The study has some limitations. The steps may not contribute equally to the proper delivery of medication; therefore the scoring may not truly denote the quality of drug delivery. Also, as the participants were already aware of having enrolled in the study, the technique demonstrated by them in the outpatient department may not be representative of the method usually followed at home. In addition, being conducted in a single centre, the study results cannot be generalised to the entire nation. The study population could be seen as insufficiently heterogeneous. Furthermore, despite being an academic centre, the inhalation technique of patients is still largely unsatisfactory. It might be assumed that the correct inhalation technique in non-academic centres and general practice clinics without face-to-face training is less satisfactory. Lastly, our study is limited to evaluation of dry power inhalers, which were commonly used in our region owing to its low price and relatively easy usage.

\section{Conclusion}

Inhalation technique is mostly unsatisfactory, especially in older patients with low education level and severe obstruction. Regular review and reinforcement of the correct inhalation method is essential for effective inhaler use and better symptom control.

\section{References}

1 Global Initiative for Asthma (GINA). Global strategy for asthma management and prevention. GINA, 2008. www.ginasthma.org [Accessed 1 December 2018].

2 Brocklebank D, Ram F, Wright J et al. Comparison of the effectiveness of inhaler devices in asthma and chronic obstructive airways disease: a systematic review of the literature. Health Technol Assess 2001:5:1-149.
3 Lavorini F, Magnan A, Dubus JC et al. Effect of incorrect use of dry powder inhalers on management of patients with asthma and COPD. Respir Med 2008;102:593-604.

4 Ganguly A, Das AK, Roy A et al. Study of proper use of inhalational devices by bronchial asthma or COPD patients attending a tertiary care hospital. J ClinDiag Res 2014;8:4-7.

5 Arora P, Kumar L, Vohra V et al. Evaluating the technique of using inhalation device in COPD and bronchial asthma patients. Respir Med 2014;8:992-8.

6 Jarvis S, Ind PW, Shiner RJ. Inhaled therapy in elderly COPD patients; time for re-evaluation? Age and Ageing 2007;36:213-8.

7 Rootmensen GN, Van Keimpema AR, Jansen HM, de Haan RJ. Predictors of incorrect inhalation technique in patients with asthma or COPD: A study using a validated videotaped scoring method. J Aerosol Med Pulm Drug Deliv 2010;5:323-8.

8 Goodman DE, Israel E, Rosenberg M, Johnston R, Weiss ST, Drazen $\mathrm{JM}$. The influence of age, diagnosis, and gender on proper use of metered-dose inhalers. Am J Respir Crit 1994;150:1256-61.

9 Sapkota D, Amatya YR. Assessment of rotahaler inhalation technique among patients with chronic obstructive pulmonary disease in a teaching hospital in Nepal. Journal of Kathmandu Medical College 2017:5:11-7.

10 Rau JL. Practical problems with aerosol therapy in COPD. Respir Care 2006:51:158-72.

11 Carrion VF, Maya MM, Fontana SI, Diaz LJ, Marin PJ. Inhalation technique in patients with chronic respiratory diseases. Archivos De Bronconeumologia 2000;36:236-40.

12 Plaza V, Sanchis J. Medical personnel and patient skill in the use of metered dose inhalers: a multicentric study. CESEA Group. Respiration 1998:65:195-8.

13 Crompton GK, Barnes PJ, Broeders $\mathrm{M}$ et al. The need to improve inhalation technique in Europe: a report from the Aerosol Drug Management Improvement Team. Respir Medicine 2006;100:1479-94. 